\title{
RELATO DE CASO: DISSECÇÃO AÓRTICA PROXIMAL EM AVESTRUZ (STRUTHIO CAMELUS)
}

TERRA, Sílvia de Azevedo ${ }^{1}$

CAMPOS, Aline Gomes ${ }^{2}$

SILVA, R. A. ${ }^{2}$

REIS, M. A. ${ }^{3}$

TEIXEIRA, V. P. A. ${ }^{3}$

Recebido em: 2009-04-01

Aprovado em: 2009-04-29

Issue DOI: $10.3738 / 1982.2278 .193$

RESUMO Um avestruz fêmea, vítima de morte súbita, foi submetido à necropsia em que foram observados, macroscopicamente, hematoma na cavidade pericárdica, hipertrofia concêntrica do ventrículo esquerdo, hematoma intramural dissecante da aorta proximal e hemorragia pulmonar. Após estudos histopatológicos e dosagem da concentração do cobre hepático, considerou-se a hipertensão arterial como principal fator de risco para a dissecção aórtica.

Palavras -chave: Avestruz. Dissecção aórtica. Hipertensão. Cobre.

\section{CASE REPORT: PROXIMAL AORTIC DISSECTION IN OSTRICH (Struthio camelus)}

\begin{abstract}
A female ostrich died suddenly was submitted to the autopsy and the gross lesions observed were hematoma in the pericardiac cavity, concentrical hypertrophy of the left ventricle, dissecting aortic aneurysm of thoracic segment and pulmonary hemorrhage. After histopathologic studies and hepatic cooper concentration, we had consider arterial hypertension as the major factor of risk to the aortic dissection.
\end{abstract}

Keywords: Ostrich. Aortic dissection. Hypertension. Cooper.

\section{INTRODUÇÃO}

A dissecção aórtica é uma condição grave que, entre animais, é constante causa de morte súbita. Na maioria das vezes, as dissecções aórticas iniciam-se através de uma lesão

\footnotetext{
1 Professora Doutora, Departamento de Morfologia, Faculdade Doutor Francisco Maeda -

Fundação Educacional de Ituverava (FAFRAM/FE), Ituverava/SP Endereço para correspondência : Rod. Jerônimo Nunes

Macedo - Km 01 - Ituverava - SP. Cep: 14.500-000. Tel. (16) 3839-6014 - satgcj@hotmail.com

2 Acadêmica do Curso de Ciências Biológicas, FAFRAM/FE

3 Professor(a) Doutor(a), Departamento de Ciências Biológicas, Universidade Federal do Triângulo Mineiro, Ubera$\mathrm{ba} / \mathrm{MG}$
} 
intimal prévia, através da qual o sangue penetra e percorre por entre os planos laminares da camada média em sentido distal, formando um falso lúmen preenchido por sangue que, quase invariavelmente, sofre ruptura provocando uma hemorragia maciça (SCHOEN, 2000; SAWHNEY et al., 2009). O termo hematoma aórtico dissecante, uma variação da dissecção aórtica, tem sido preferido ao aneurisma devido ao fato de não haver dilatação da parede da aorta, como ocorre nos aneurismas verdadeiros (BOULINEAU et al., 2005). No entanto, o termo hematoma intramural dissecante tem sido utilizado, entre os casos humanos, quando a hemorragia ocorre dentro da parede da aorta na ausência de lesões intimais e decorrentes provavelmente de alterações degenerativas da camada média predispondo à ruptura dos vasa vasorum (SAWHNEY et al., 2009).

As causas mais comuns da dissecção aórtica incluem hipertensão (SCHOEN, 2000), alterações do desenvolvimento (congênitas ou hereditárias), degenerativas, nutricionais, inflamatórias ou arterioscleróticas (JONES et al., 2001). A dissecção aórtica tem sido relatada com relativa freqüência em humanos idosos com hipertensão (SAWHNEY et al., 2009), mas em animais há poucos relatos descritos em bovinos, eqüinos, suínos, cães e aves (VAN VLEEN; FERRANS, 1998; JONES et al., 2000; BOULINEAU et al., 2005). As espécies de aves acometidas têm sido perus e avestruzes (MITCHINSON; KEYMER, 1977; VANHOOSER et al., 1994; FERRERAS et al., 2001), sendo destacada como principal causa a nutricional. O objetivo deste trabalho foi descrever os achados anatomopatológicos encontrados em avestruz vítima de morte súbita por dissecção aórtica.

\section{HISTÓRIA DO CASO E RESULTADOS}

Um avestruz fêmea, de um ano e meio, mantido em piquete de pasto comum, próximo a criações de ovinos e bovinos, alimentando-se de ração comercial formulada para avestruzes, sofreu morte súbita (semelhante ao que já havia ocorrido com outros dois avestruzes na mesma propriedade) e foi encaminhado ao setor de Anatomia Patológica da Faculdade de Medicina Veterinária Dr. Francisco Maeda onde foi submetido à necropsia.

A necropsia revelou tamponamento pericárdico, hematoma mediastinal envolvendo também o pericárdio parietal e os vasos da base do coração. A artéria aorta apresentou dorsalmente, na região do arco aórtico, uma abertura de aproximadamente $0,8 \mathrm{~cm}$ decorrente de sua ruptura. Assim a dissecção inciou-se no arco aórtico, com perfuração da parede a este nível, estendeu-se ao longo da camada média e restabeleceu comunicação com o lúmen aórtico ao nível do mediastino médio. O hematoma intramural dissecante formado na aorta proximal caracterizou-se pela dissecação da parede separando a camada íntima da média e as várias camadas desta última, em áreas livres de arteriosclerose aterosclerótica, estendendo-se desde a base do coração por, aproximadamente, $6 \mathrm{~cm}$ ao longo da aorta torácica, além do arco aórtico (Figura 1). Evidenciou-se, ainda, extensa hemorragia torácica, hipertrofia concêntrica do 
ventrículo esquerdo (Figura 2), esteatose hepática difusa e moderada. A artéria aorta foi toda analisada, mas não foram encontradas outras alterações em seus ramos.

Fragmentos coletados dos tecidos foram fixados em solução de formalina tamponada a $10 \%$, processados, emblocados em parafina e cortes de $0,5 \mu \mathrm{m}$ foram corados pela hematoxilionaeosina (HE). Microscopicamente, evidenciou-se hemorragia entre as camadas de fibras elásticas e leiomiócitos aórticos, no interior dos alvéolos pulmonares, associada a áreas de colapso pulmonar, e, ainda, hialinose e necrose fibrinóide em arteríolas renais (Figura 3).

Fragmentos hepáticos fixados foram processados para extração do cobre em ácido nítrico concentrado e, através de espectrofotometria por absorção atômica, foi realizada a dosagem da concentração do cobre hepático, a qual foi de 6,67 ppm.

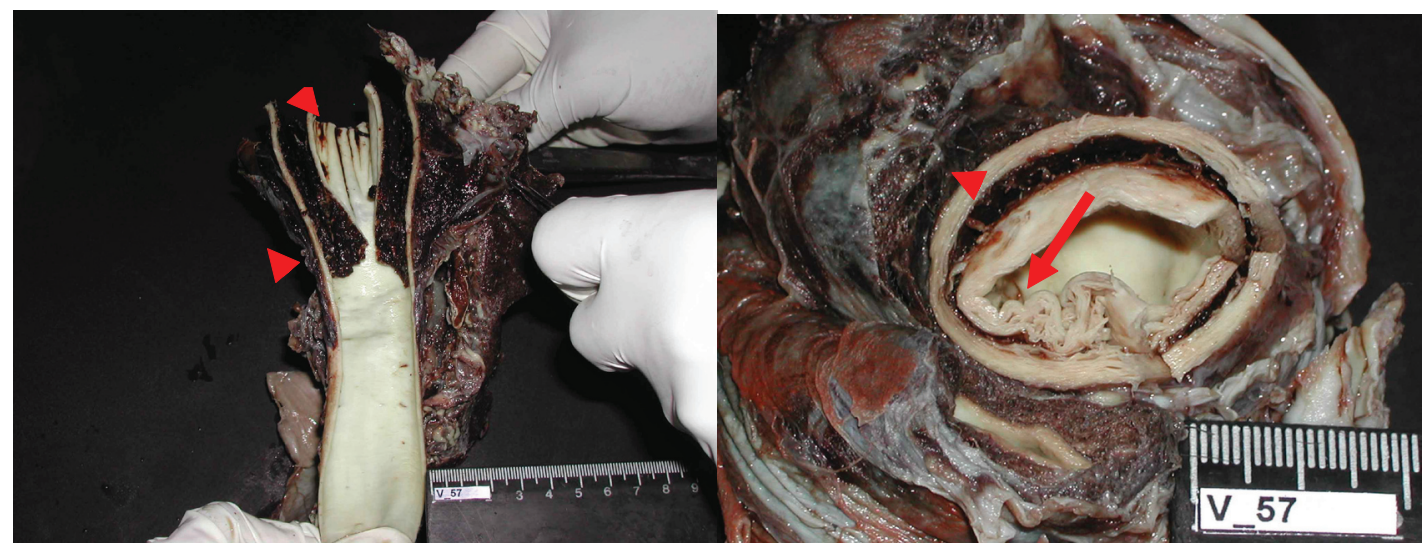

FIGURA 1: Aspecto macroscópico de dissecção aórtica proximal em avestruz. Em A - Segmento torácico da artéria aorta de avestruz com hematoma intramural dissecante (cabeças de seta). Em B- Corte transversal da artéria aorta na base do coração. Dissecção das camadas da artéria em vários folhetos (seta), hematoma intramural dissecante (cabeça de seta)

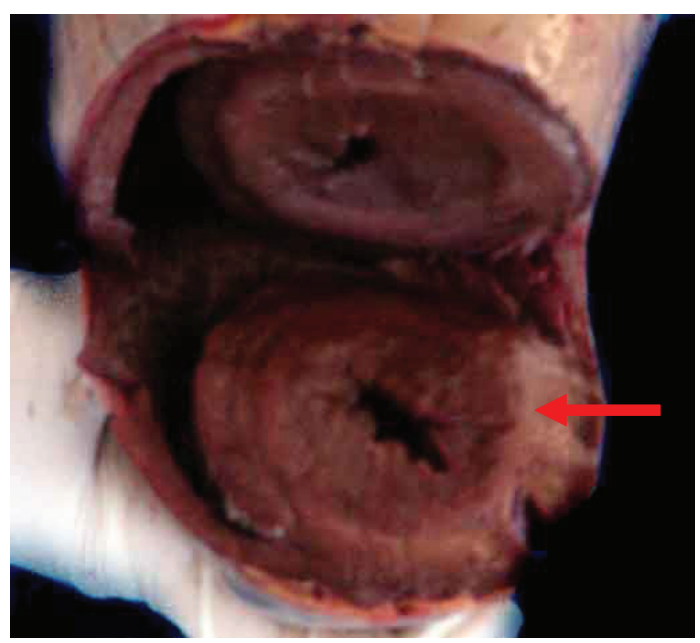

FIGURA 2: Corte transversal de coração de avestruz. Hipertrofia concêntrica do ventrículo esquerdo (seta). 


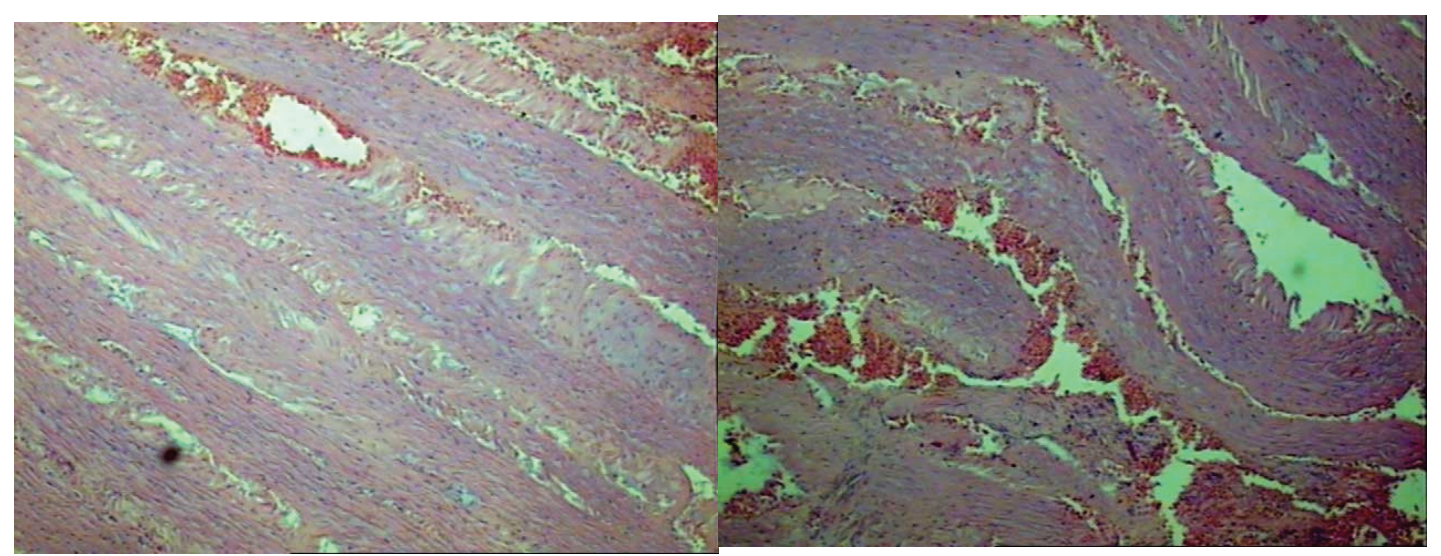

FIGURA 3 : Aspecto microscópico de hematoma intramural dissecante da aorta proximal em avestruz. Em A Aorta torácica. Camada média da aorta com hemácias e fibrina separando as fibras elásticas e leiomiócitos, 400X, HE (seta). Em B - Aorta ascendente. Dissecção da camada média por hemácias e fibrina, 600X, HE (seta).

\section{DISCUSSÃO}

No caso deste avestruz, a característica hipertrofia concêntrica do ventrículo esquerdo possivelmente foi causada por hipertensão arterial, a qual pode ter sido decorrente de condições de estresse às quais o animal possa ter sido submetido, uma vez que ele era mantido em piquetes próximo a outras espécies animais. A hipertensão provavelmente propiciou a ruptura da camada íntima da aorta e, conseqüentemente, o desenvolvimento do hematoma intramural dissecante, bem como hematoma na cavidade pericárdica e a maciça hemorragia pulmonar que culminaram com as causas de morte do animal - tamponamento cardíaco e insuficiência respiratória, respectivamente.

Em primatas (BOORMAN et al., 1976; SCHOEN; COTRAN, 2000), cães e gatos (JONES et al., 2000; BOULINEAU et al., 2005), a hipertensão arterial e arteriosclerose aterosclerótica se destacam entre os principais fatores de riscos para dissecção da aorta, porém em aves existem poucos relatos que relacionam o aneurisma à hipertensão (FERRERAS et al., 2001). Este caso apresentou alterações macroscópicas como a hipertrofia cardíaca esquerda concêntrica e as alterações microscópicas como hialinose e necrose fibrinóide em arteríolas renais, compatíveis com hipertensão arterial.

A deficiência de cobre, a qual gera alterações nas ligações entre as fibras elásticas e colágenas da parede vascular, juntamente com outros fatores de risco como hipertensão arterial (provavelmente de corrente de condições de estresse), pode ser implicada na patogênese do aneurisma dissecante da aorta. Em perus, suínos e ratos (VANHOOSER et al., 1994; JONES et al., 2000; FERRERAS et al., 2001), existem evidências de que a deficiência nutricional de cobre resulta na inadequada formação da parede dos vasos, uma vez que a ligação entre fibras elásticas e colágenas da parede vascular depende da enzima lisil oxidase a qual é dependente de cobre (BAUER, 1992; VAN VEEN, 1999; HRISTOVA; HENRY, 2001). Dessa forma, uma deficiência de cobre poderia tornar a parede da aorta mais sensível a variações de pressão. No 
entanto, no neste caso, a concentração hepática de cobre deste animal foi dentro dos parâmetros normais (4-9 ppm) (PULS, 1994) e a ração consumida pelo animal era utilizada comercialmente, o que nos leva a crer que os níveis de cobre não foram os fatores determinante do aneurisma.

\section{CONCLUSÃO}

O avestruz deste caso não apresentou deficiência nos níveis hepáticos de cobre, assim a causa provável da dissecção aórtica proximal com formação de hematoma intramural dissecante foi a hipertensão arterial, possivelmente decorrente do estresse causado pela convivência com outras espécies animais, como ovinos e bovinos no mesmo piquete. No entanto, como houve relatos de morte súbita em outros dois animais (não pesquisados), uma outra causa que poderia ser levantada seria alguma alteração do desenvolvimento da parede aórtica.

\section{AGRADECIMENTOS}

Os autores agradecem aos funcionários do laboratório de solos da Faculdade Dr. Francisco Maeda (FAFRAM) e do laboratório de histopatologia da Disciplina de Patologia Geral da Universidade Federal do Triângulo Mineiro (UFTM) pelo auxílio no preparo do material pesquisado.

\section{REFERÊNCIAS}

BAUER, J. D. Inorganic elements and blood gases. In: Clinical laboratory methods.

9. ed. Saint Louis: Mosby Company, 1982. chap. 22, p. 506-534.

BOORMAN, G. A.; SILVERMAN, S.; ANDERSON, J. H. Spontaneous dissecting aortic aneurysm in a squirrel monkey (Simiri sciureus). Laboratory Animal Sciences, v. 26, p. 942947, 1976.

BOULINEAU, T. M.; ANDREWS-JONES, L.; VANALSTINE, W. Spontaneus aortic dissecting hematoma in two dogs. Journal of Veterinary Diagnostic Investigation, v. 17, p. 492-497, 2005.

FERrERAS, M. C.; GONZÁLEZ, J.; PÉREZ, V.; REYES, L. E.; GÓMEZ, N.; PÉREZ, C.; CORPA, J. M.; GARCÍA-MARIN, J. F. Proximal aortic dissection (dissecting aortic aneurysm) in a mature ostrich. Avian Diseases, v. 45, p. 251-256, 2001.

HRISTOVA, E. N.; HENRY, J. B. Metabolic intermediates inorganic ions and biochemical 
markers of bone metabolism. In: HENRY, J. B. (ed). Clinical diagnosis and management by laboratory methods. 20. ed. Philadelphia: Saunders company, 2001. chap. 10, p. 180-210.

JONES, T. C.; HUNT, R. D.; KING, N. W. Sistema Cardiovascular. In: . Patologia Veterinária. 6. ed. São Paulo: Manole, 2000. cap. 21, p. 991-1026.

MITCHINSON, M. J.; KEYMER, I. F. Aortic rupture in ostriches (Struthio camelus) - a comparative study. Journal of Comparative Pathology, v. 87, p. 27-33, 1977.

PULS, R. Mineral levels in animal healthy: diagnostic data. 2. ed. Sherpa International, 1994. $250 \mathrm{p}$.

SAWHNEY, N. S.; DEMARIA, A. N.; BLANCHARD, D. G. Aortic intramural hematoma - an increasingly recognized and potentially fatal entity. Chest, v. 120, p. 1340-1346, 2001.

SCHOEN, F. J.; COTRAN, R. S. Vasos sanguíneos. In: COTRAN, R. S.; KUMAR, V.; COLLINS, T. Robbins - Patologia Estrutural e Funcional. 6. ed. Rio de Janeiro: Guanabara Koogan, 2000. cap. 12, p. 441-485.

VAN VEEN, L. Aortic rupture in a poultry: a review. Tijdschrift voor Diergeneeskunde, v. 124, n. 8 , p. 244-247.

VAN VLEET, J. F.; FERRANS, V. J. Patologia do sistema cardiovascular. In: CARLTON, W. W.; MACGAVIN, M. D. Patologia Veterinária Especial de Thomson. 2. ed. Porto Alegre: Artmed, 1998. cap. 4, p. 194-227.

VANHOOSER, S. L.; STAIR, E.; EDWARDS, W. C. Aortic rupture in ostrich associated with cooper deficiency. Veterinary and Human Toxicology, v. 36, p. 226-227, 1994. 